\title{
Community Services, Progression Rate, and Nursing Home Placement In Persons with Alzheimer's Disease
}

\author{
Jocelyn Shealy McGee, Dennis R. Myers, Haley Groce \\ Garland School of Social Work, Baylor University, Waco, Texas, USA \\ Email address: \\ jocelyn_mcgee $a$ baylor.edu (J. S. McGee), dennis_myers $a$ baylor.edu (D. R. Myers)
}

\section{To cite this article:}

Jocelyn Shealy McGee, Dennis R. Myers, Haley Groce. Community Services, Progression Rate, and Nursing Home Placement in Persons with Alzheimer's Disease. American Journal of Psychiatry and Neuroscience. Vol. 7, No. 1, 2019, pp. 12-18.

doi: 10.11648/j.ajpn.20190701.13

Received: March 22, 2019; Accepted: May 15, 2019; Published: June 12, 2019

\begin{abstract}
There are many factors influencing when a person with Alzheimer's disease (AD) or another dementia is placed in a nursing home (NHP). The aims of this study were to determine if introducing home and community-based services (HCBS) at different stages of disease severity influenced time to NHP in a sample of persons living with AD $(n=1210)$; examine if disease pre-progression rate (PPR) influenced time to NHP; and explore the interaction between disease severity at first use of HCBS and PPR on time to NHP. Findings suggested that earlier introduction of HCBS delayed time to NHP in a univariate model $(\mathrm{p}=0.03)$, but not when covariates were adjusted $(\mathrm{p}=0.39)$; faster PPR resulted in earlier NHP in both univariate $(\mathrm{p}<$ $0.001)$ and covariate adjusted models $(\mathrm{p}<0.01)$; and earlier introduction of HCBS delayed time to NHP (even when PPR was taken into consideration). Implications are discussed.
\end{abstract}

Keywords: Dementia, Alzheimer's, Nursing Home Placement, Community Services

\section{Introduction}

An estimated 5.7 million people in the United States of America (USA) and 50 million people worldwide are living with Alzheimer's disease (AD) and other age-related dementias. [1-2] These numbers are expected to rise dramatically by the year 2050 to 14 million in the USA and 152 million worldwide. [1-2] The financial costs of care for this population in the USA was approximately \$277 billion in 2018; including \$186 billion in Medicare and Medicaid payments. [1] Worldwide, this figure translates to $\$ 1$ trillion dollars in 2018; which is expected to rise to $\$ 2$ trillion dollars by the year 2030. [2] For one year of nursing home care in the USA, it costs approximately $\$ 89,300$ for a person living with dementia - as compared to $\$ 18,720$ per year for adult day health care services. [3]

Many persons with $\mathrm{AD}$ have not made sufficient plans for formal long-term support and plan to rely on family caregivers in the home. [4] However, their needs may surpass what their families are able to provide increasing the likelihood of NHP as compared to older adults who do not have dementia. [1, 5] Indeed, an estimated $75 \%$ of persons living with dementia over age 80 reside in nursing homes as compared to only $4 \%$ of those who do not have dementia. [6]

The decision to place a loved one in a nursing home is determined by a complex array of factors [7-12] related to the individual with dementia and their family members. The most consistent risk factors include severity of cognitive impairment, functional dependencies, behavioral problems, depression, and sociodemographic indicators such as lower annual income and Caucasian race/ethnicity. [7, 13-18] Also, the degree of emotional distress experienced by family members is a risk factor for placement. [19, 20]

Of concern, people with dementia who are placed in nursing homes have high mortality rates, ranging between $25.7 \%$ and $29.9 \%$, in the first year after placement. [21-22] Those with more advanced dementia live on average 173.7 days from time of NHP to death [23] Almost $66 \%$ of persons living with dementia die in nursing homes, compared to just $20 \%$ of people dying with cancer and $28 \%$ dying from all other conditions ${ }^{1}$ even though the preference of most persons with dementia is to die in their own home. [24] 
Home and Community Based Services (HCBS) are interventions aimed at helping persons with dementia live in their home for as long as possible by providing respite and relief to informal caregivers. [25-27] Broadly defined, HCBS entails the use of a hired worker to assist with tasks such as housework, limited medical care, mobility, and/or companionship to a family caring for a person with dementia. [26] Out-of-home services, such as adult day programs, provide therapeutic activities, social activities, and health monitoring for persons with dementia at an institution on a daily to weekly basis. [28] Some studies suggest that introducing HCBS earlier in the course of dementia may reduce the likelihood of $\mathrm{NHP}^{28}$ such as the use of paid caregiver services or adult day services [2930].

There is evidence that informal caregivers tend to utilize HCBS in the later stages of disease progression rather than earlier in the course of the disease. [26, 31] Some factors contributing to this trend are a lack of awareness of the services available, family caregivers' feeling guilty about giving up the care of a relative to others, and/ or the cost of services. [26] Informal caregivers who make use of adult day services are more likely to utilize other HCBS due to their awareness of such supports according to some studies. [30]

In light of previous research on factors influencing time to NHP in people with $\mathrm{AD}$, we sought to determine if introducing home and HCBS at different stages of disease severity influenced time to NHP in a sample of persons living with $\mathrm{AD}(\mathrm{n}=1210)$; examine if disease pre-progression rate (PPR) influenced time to NHP; and explore the interaction between disease severity at first use of HCBS and PPR on time to NHP.

\section{Methods}

This secondary data analysis was approved by the Baylor College of Medicine Institutional Review Board.

\subsection{Participants}

Data was retrieved from a longitudinal database at the Baylor College of Medicine's Alzheimer's Disease and Memory Disorders Center (BCM ADMDC). Participants were diagnosed using the National Institute of Neurological and Communicative Disorders and Stroke (NCDS) and the Alzheimer's Disease and Related Disorders Association (ADRDA) criteria. [32] In addition to neurological, neuropsychological, and other medical assessments, they received a questionnaire on their use of HCBS (i. e., companion/sitter in the home, supervision by housekeeper in the home and adult day health care) and whether or not they have been placed in a nursing home in any given year.

The final sample consisted of 1210 participants. In regards to disease severity, 381 participants were rated as mild ( $M_{\text {age }}$ $=74,38.6 \%$ female), 414 as moderate $\left(M_{\text {age }}=75,30.4 \%\right.$ female), and 356 as severe ( $M_{\text {age }}=71$ years, $27.3 \%$ female). Detailed demographic information and other relevant data about the sample are depicted in Table 1.

\subsection{Measures}

\subsubsection{Pre-progression Rate (PPR)}

Formula (1) for PPR was developed by Doody, et al. as a predictor of the rate of cognitive decline over time. [33] It is calculated as follows:

Maximum Possible MMSE Score-Baseline MMSE Score Physician's Estimate of AD Duration

The maximum possible Mini Mental Status Exam (MMSE) score is the highest score a participant can achieve. [34] Baseline MMSE score is the score received by the participant at initial presentation. Duration of $\mathrm{AD}$ is estimated according to a standardized BCM ADMDC protocol which includes a series of questions about the duration of specific symptoms, combined with a review of medical records, an informant interview, and hypothesis-testing. This method for estimating disease duration predicts onset of AD to the nearest half-year. [35] From this score, participants can be categorized as slow (0-1.9 MMSE points/ year), intermediate (2-4.9 MMSE points/year), or rapid progressors (5 or more MMSE points/ year) per Doody et. al. [33]

\subsubsection{HCBS Use}

Use of HCBS was assessed with a questionnaire distributed prior to the initial visit and then again at each annual follow-up visit (with a fixed set of options to identify the services used within the past year). In this study, HCBS included home-based care (defined as the help of a hired worker to serve as a companion, homemaker, home-health aid, or nurse in the patient's home), and adult day health care (defined as out-of-home day services such as therapeutic activities, social activities, and health monitoring).

\subsubsection{Severity of AD at First HCBS Use}

Severity of AD at first HCBS use was determined by using an individual's score on the MMSE at the time HCBS was first used. Participants were then classified as having mild (20-30 points), moderate (11-19), or severe AD. [1-10,34]

\subsubsection{Time to NHP}

The dependent variable was calculated as time in months from the physician-estimated onset of disease to the date a participant moved to a nursing home.

\subsubsection{Covariates}

Factors used as covariates in this analysis were age, sex, years of education, functional status (based on Physical SelfMaintenance Scale [PSMS] and Instrumental Activities of Daily Living [IADL] scores), [36] caregiver age, caregiver relationship to participant, and level of caregiver stress (measured by asking caregivers to rate their level of stress in caring for the person with $\mathrm{AD}$ on a 4-point Likert-type scale ( $1=$ lowest stress; 4 = highest stress $)$. See Table 1 . 
Table 1. Key baseline demographic and predictive characteristics by severity at HCBS introduction.

\begin{tabular}{|c|c|c|c|}
\hline Baseline Variable & Mild Stage $(n=381)$ & Moderate Stage $(n=414)$ & Severe Stage $(n=356)$ \\
\hline Participant age, $\mathrm{y}$, mean* & 74 & 75 & 71 \\
\hline Participant female* & $38.60 \%$ & $30.40 \%$ & $27.30 \%$ \\
\hline \multicolumn{4}{|l|}{ MMSE* } \\
\hline Mild (20-30) & $95.00 \%$ & $48.30 \%$ & $30.50 \%$ \\
\hline Moderate (11-19) & $5.00 \%$ & $51.50 \%$ & $33.60 \%$ \\
\hline Severe $(0-10)$ & $0.00 \%$ & $0.30 \%$ & $35.90 \%$ \\
\hline IADL, mean* & 12.62 & 16.17 & 18.87 \\
\hline \multicolumn{4}{|l|}{ PPR*(MMSE points/ year) } \\
\hline Slow $(0-1.9)$ & $49.00 \%$ & $30.70 \%$ & $14.70 \%$ \\
\hline Intermediate $(2-4.9)$ & $39.20 \%$ & $49.50 \%$ & $49.20 \%$ \\
\hline Rapid $(\geq 5)$ & $11.80 \%$ & $31.90 \%$ & $36.20 \%$ \\
\hline Duration of symptoms, $y$, mean* & 3.21 & 3.92 & 4.12 \\
\hline Caregiver age, y & 63 & 62 & 61.57 \\
\hline \multicolumn{4}{|l|}{ Caregiver relationship to participant } \\
\hline Other & $13.60 \%$ & $16.00 \%$ & $16.60 \%$ \\
\hline Caregiver stress level, mean & 2.71 & 2.79 & 2.86 \\
\hline NHP, $n, \% *$ & $31(9.69 \%)$ & $48(12.87 \%)$ & $77(24.29 \%)$ \\
\hline
\end{tabular}

$* \mathrm{p}<0.05$.

\section{Analysis}

Demographic and clinical variables were compared based on the disease stage at which HCBS was first introduced using chi-squared tests (discrete variables) or ANOVA (continuous variables). Analysis was conducted using SAS JMP 11 Pro. [37] A univariate Cox proportional hazards regression model and $\log$ rank test were used to determine the unadjusted effect of: a) stage of severity at first use of
HCBS; b) PPR; and c) the interaction between severity stage at first use of HCBS and PPR on time to NHP. Kaplan-Meier curves were presented to compare time to NHP between participants who first used HCBS in the mild, moderate, or severe stages of $\mathrm{AD}$ and participants with slow, intermediate, or rapid PPRs. A multivariate Cox proportional hazards regression model was used to determine these associations while controlling for covariates. Results of the analysis are depicted in Tables 2 and 3; and Figures 1 and 2.

Table 2. Unadjusted effects of disease severity at first use of HCBS, PPR, and their interaction on time to NHP.

\begin{tabular}{llll}
\hline Variable & HR & CI & P \\
\hline Severity Stage at First use of HCBS & & & 0.03 \\
Mild vs. Severe & 0.60 & $0.32,1.16$ & 0.12 \\
Moderate vs. Severe & 0.44 & $0.23,0.83$ & 0.01 \\
Pre-progression Rate & & & $<0.001$ \\
Intermediate vs. Slow & 3.04 & $1.58,5.85$ & $<0.001$ \\
Rapid vs. Slow & 6.00 & $2.53,14.23$ & 0.001 \\
Interaction (Severity at HCBS first use x PPR) & & & 0.21 \\
Severe Stage & 1.38 & $0.68,2.79$ & 0.37 \\
Intermediate vs. Slow & 2.31 & $0.97,5.52$ & 0.06 \\
Rapid vs. Slow & 0.60 & $0.29,1.22$ & 0.16 \\
Intermediate vs. Rapid & & & 0.01 \\
Moderate Stage & 4.53 & $1.48,13.84$ & $<0.001$ \\
Intermediate vs. Slow & 11.80 & $3.30,42.26$ & 0.05 \\
Rapid vs. Slow & 0.38 & $0.15,1.01$ & 0.03 \\
Intermediate vs. Rapid & & & 0.003 \\
Mild Stage & 3.05 & $1.14,8.18$ & 0.17 \\
Intermediate vs. Slow & 7.85 & $2.05,30.04$ & $0.10,1.47$ \\
Rapid vs. Slow & 0.39 & & \\
Intermediate vs. Rapid & & & \\
\hline
\end{tabular}

Table 3. Adjusted effects of disease severity at first use of HCBS, PPR on time to NHP.

\begin{tabular}{lccc}
\hline Variable & HR & CI & P \\
\hline Participant Variables & & & 0.39 \\
Stage at first use of HCBS & & & 0.68 \\
Mild vs. Severe & 1.30 & $0.38,4.40$ & 0.36 \\
Moderate vs. Severe & 0.66 & $0.24,1.78$ & 0.20 \\
Mild vs. Moderate & 1.98 & $0.70,5.60$ & \\
\hline
\end{tabular}




\begin{tabular}{|c|c|c|c|}
\hline Variable & HR & CI & $\mathbf{P}$ \\
\hline Pre-progression Rate & & & $<0.01$ \\
\hline Intermediate vs. Rapid & 0.49 & $0.17,1.44$ & 0.19 \\
\hline Intermediate vs. Slow & 4.30 & $1.53,11.88$ & 0.005 \\
\hline Rapid vs. Slow & 8.60 & $2.04,36.28$ & 0.003 \\
\hline Age & 0.95 & $0.90,1.0$ & 0.05 \\
\hline Sex (male vs. female) & 1.91 & $0.78,4.70$ & 0.16 \\
\hline Education & 0.95 & $0.85,1.06$ & 0.38 \\
\hline Baseline MMSE score & 1.11 & $1.02,1.21$ & 0.01 \\
\hline Baseline IADL score & 1.08 & $0.98,1.19$ & 0.14 \\
\hline Baseline PSMS score & 1.12 & $1.0,1.25$ & 0.05 \\
\hline \multicolumn{4}{|l|}{ Caregiver Variables } \\
\hline Age & 0.98 & $0.94,1.02$ & 0.31 \\
\hline Relationship to participant & & & 0.05 \\
\hline Spouse vs. Adult-Child & & $0.05,0.74$ & \\
\hline Spouse vs. Other & & $0.09,1.47$ & \\
\hline Adult-Child vs. Other & & $0.55,6.11$ & \\
\hline Baseline stress & & & 0.56 \\
\hline "Just a Little" vs. "Extreme" & & $0.47,3.53$ & \\
\hline "Moderate" vs. "Extreme" & & $0.34,1.87$ & \\
\hline "Just a Little" vs. "Moderate" & & $0.66,3.98$ & \\
\hline
\end{tabular}

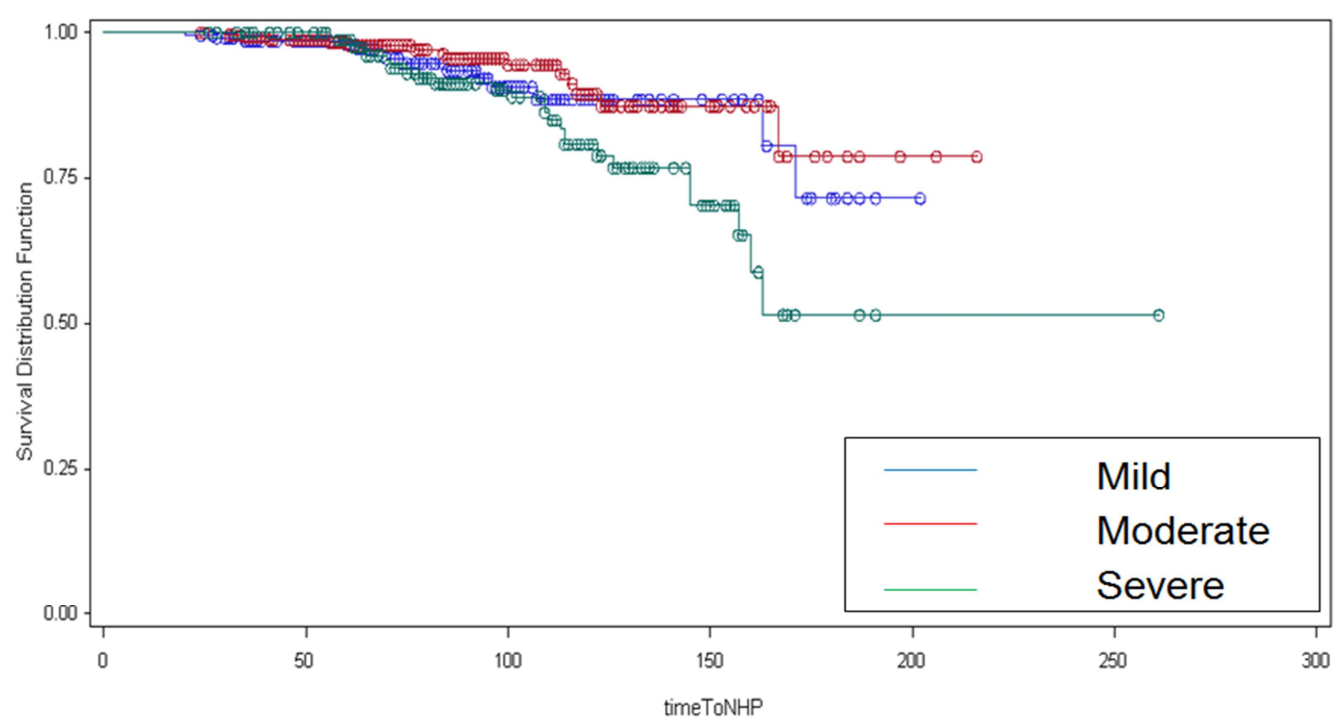

Figure 1. Kaplan-Meier survival curves for time to NHP by disease severity when HCBS was introduced.

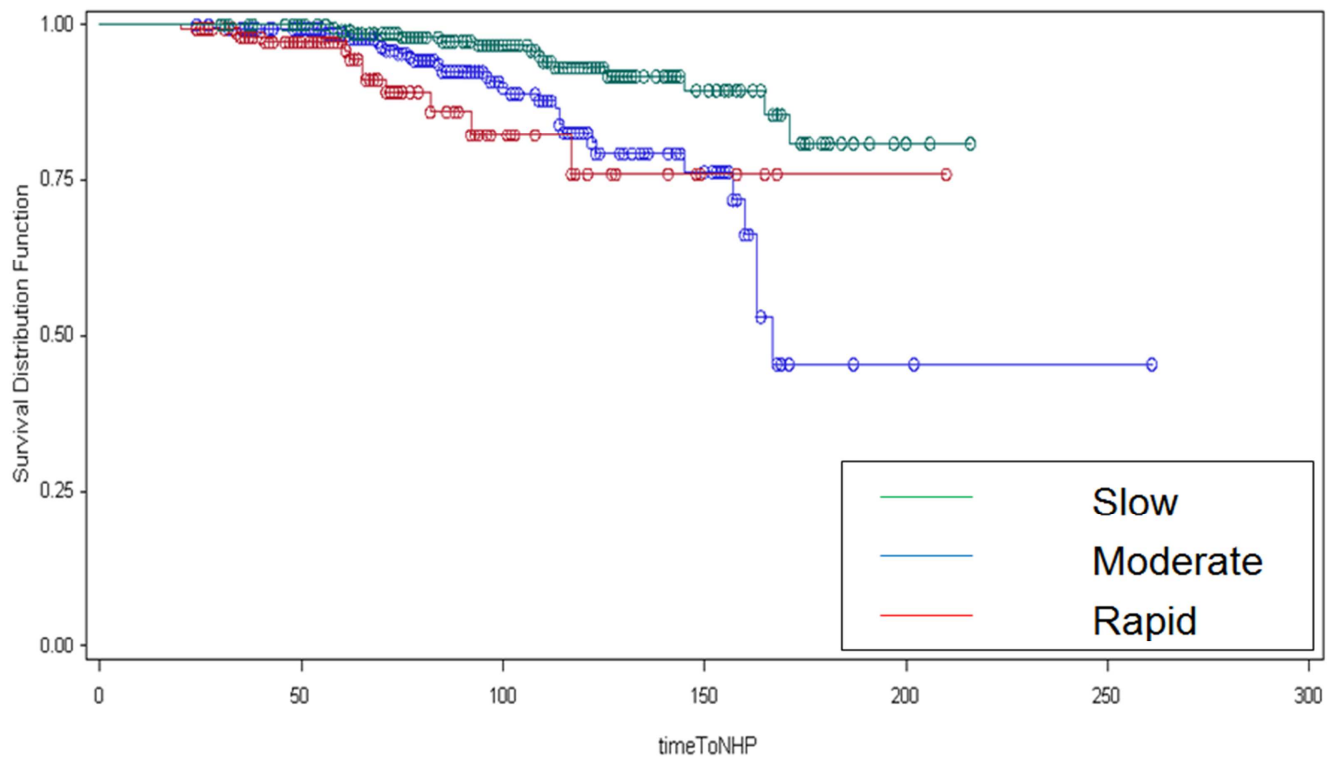

Figure 2. Kaplan-Meier illustrating time to NHP by pre-progression rate. 


\section{Results}

The first aim of this study was to determine if introducing home and community-based services (HCBS) at different stages of disease severity influenced time to NHP. Findings confirmed that starting HCBS earlier in the disease progression (e. g., at an earlier stage) delayed time to NHP in the univariate analysis $(p=0.03)$. However, this association was not present after adjusting for covariates $(p=0.39)$. As expected, the group who first used HCBS in the severe stage were placed more quickly than those who started using services in the mild stage ( $H R$ for mild vs. severe $=0.60, H R$ for moderate vs. severe $=0.44, \mathrm{X}^{2}=7.19, p=0.03$ ).

The second aim of the study was to examine the relationship between PPR and time to NHP. As expected, participants with slower PPRs were not placed as soon as participants with faster PPRs $(p<0.001)$. This finding held true after adjusting for covariates $(p<0.01)$. The two largest effects were from the adjusted intermediate verses slow PPR $(H R=4.30, p=0.005)$ and the adjusted rapid verses slow PPR $(H R=8.60, p=0.003)$. Intermediate versus rapid PPRs showed a modest and non-statistically significant ratio $(H R=$ $0.49, p=0.19)$. Those with a rapid PPR were placed sooner than those with slow a PPR ( $H R$ for intermediate vs. slow $=$ 2.64, $H R$ for rapid vs. slow $=5.47, \mathrm{X}^{2}=17.41, p=0.0002$ ).

The third aim of the study was to explore the interaction between disease severity at first use of HCBS and PPR on time to NHP. There was a significant association after adjusting for covariates which suggests that: 1) having a faster PPR modifies the effect of HCBS use on time to NHP; and 2) use of HCBS at a milder stage of disease modifies the effect of disease progression on time to NHP.

\section{Discussion}

There are several complex methods for predicting time to NHP involving analysis of electronic medical records aimed at developing risk assessment metrics. These risk assessments have shown that faster rates of cognitive decline may lead to earlier NHP placement. [39] However, these methods are not readily available to healthcare providers who are not working for large healthcare systems and are not be formally trained on their use. Thus, simple and effective measurement tools are needed to better evaluate the impact of these services.

The findings of this study suggest that PPR is a simple measure of cognitive change over time that can be used to predict time to NHP in the context of other important covariates (e. g. functional status, caregiver stress, etc.). Previous research suggests that PPR is also a predictor of developing a functional disability and mortality rates in people with AD. [40] It is possible that PPR could be used as an indicator of risk even when information about other covariates are not clear.

There is a current emphasis within the healthcare industry on developing and evaluating HCBS delivery models for high risk, high need lives including persons living with $\mathrm{AD}$ and related dementias. [41] In these models, healthcare systems recognize the substantive impact that HCBS can have on the social determinants of health in these populations. The emerging partnerships between healthcare and community-based organizations [42] may strengthen the efficacy of HCBS to prevent premature NPH. A metric such as PPR may be applied in risk assessments of these populations as the industry strives for stronger partnerships focused on better health, better care, and lower costs. These alliances may also enrich the support that HCBS can provide to family caregivers, potentially delaying their opting for NPH for their loved one.

The results of this study should not be used to determine the appropriateness of NHP for persons with AD and other dementias nor does it negate the value of this important option for persons with $\mathrm{AD}$ and their family members. The decision to seek NHP is a highly complex and individualized matter that considers many different factors such as support systems (formal and informal), caregiver stress, living preferences, finances, and access, among others.

For some, quality of life can be greatly enhanced by NHP. For instance, persons with $\mathrm{AD}$ who need intensive medical or behavioral management may benefit significantly especially when staff has received effective person-centered training to address the challenging behaviors that can accompany AD. [43] For others, the possible effects of solitary living, such as apathy and depression, could negatively alter the course of the disease, making NHP a beneficial alternative to remaining at home. [44]

The opportunity to participate in a community residential care setting with peers may allow for the opportunity for increased social interaction, activities that result in pleasurable experiences, the opportunity for new relationships, and other benefits. Additionally, NHP placement may be a lifeline when stress and fatigue are experienced by informal caregivers to the extent that both caregiver and care recipient health and safety is at risk.

There were several limitations to the measures used in this study. First, the HCBS measure was based on self-report and may have been influenced by inaccurate recall of the participants who completed it. Second, participants could only report whether or not HCBS were used (e. g., yes or no) versus how much or when services were utilized. Third, there were a limited number of HCBS listed in the measure which may not have fully accounted for the range of HCBS that are available to persons with $\mathrm{AD}$ and their family members. Fourth, the PPR measure could benefit from more rigorous validation and specification. The denominator for calculating this measure relies on physician's estimate of disease duration. Further assessment of the inter-rater reliability of the measure would lend more credence to its utility even though it was highly endorsed by Doody, et al.. [33, 45-46] Finally, the participants in this study were predominantly Caucasian and more highly educated than the general population. Thus, studies using more diverse samples should 
be conducted.

\section{Conclusions}

In conclusion, numerous factors must be taken into consideration for maximal results with any clinical intervention. The use of HCBS, as a clinical intervention for preventing premature NHP, is no different than pharmacological or other psychosocial interventions in this regard. Therefore, we advocate for research that begins to develop formulas for clinical pathways for HCBS use that take into consideration disease severity (e. g. mild, moderate, severe); rate of disease progression as predicted by PPR (e. g. slow, intermediate, rapid); specific types of $\mathrm{HCBS}$; and amount or frequency of HCBS. As such, health care professionals who serve people with $\mathrm{AD}$ and their family members could craft more nuanced evidence-based treatment plans. Future studies could investigate HCBS in more depth, including both an examination of overall time spent in HCBS and time spent in HCBS per stage of severity (e. g. how much time is spent in HCBS at mild severity levels as compared to moderate severity levels) in addition to tracking changes in the frequency of use.

\section{References}

[1] Alzheimer's Association. 2018 Alzheimer's disease facts and figures. Alzheimer's \& Dementia. 2018; 14 (3): 367-429. doi: 10.1016/j.jalz.2018.02.001.

[2] Alzheimer's Disease International. World Alzheimer report 2018. Alzheimer's Disease International. https://www.alz.co.uk/research/world-report-2018. Published September 2018.

[3] Hurd MD, Martorell P, Delavande A, Mullen KJ, Langa KM. Monetary costs of dementia in the United States. New England Journal of Medicine. 2013; 368 (14): 1326-1334. doi: 10.1056/nejmsa1204629.

[4] Robison J, Shugrue N, Fortinsky RH, Gruman C. Long-term supports and services planning for the future: implications from a statewide survey of baby boomers and older adults. The Gerontologist. 2013; 54 (2): 297-313. doi: $10.1093 /$ geront/gnt094.

[5] Eaker ED, Vierkant RA, Mickel SF. Predictors of nursing home admission and/or death in incident Alzheimer's disease and other dementia cases compared to controls. Journal of Clinical Epidemiology. 2002; 55 (5): 462-468. doi: 10.1016/s0895-4356 (01) 00498-x.

[6] Callahan CM, Arling G, Tu W, et al. Transitions in care for older adults with and without dementia. Journal of the American Geriatrics Society. 2012; 60 (5): 813-820. doi: 10.1111/j.1532-5415.2012.03905.x.

[7] Gaugler JE, Duval S, Anderson KA, Kane RL. Predicting nursing home admission in the U. S: a meta-analysis. $B M C$ Geriatrics. 2007; 7 (1): 1-14. doi: 10.1186/1471-2318-7-13.

[8] Gaugler JE, Yu F, Krichbaum K, Wyman JF. Predictors of nursing home admission for persons with dementia. Medical $\begin{array}{llll}\text { Care. 2009; } 47 & \text { (2): } 191-198 . & \end{array}$ 10.1097/mlr.0b013e31818457ce.

[9] Miller EA, Weissert WG. Predicting elderly people's risk for nursing home placement, hospitalization, functional impairment, and mortality: a synthesis. Medical Care Research and Review. 2000; 57 (3): 259-297. doi: $10.1177 / 107755870005700301$.

[10] Nygaard HA, Albrektsen G. Risk factors for admission to a nursing home: a study of elderly people receiving home nursing. Scandinavian Journal of Primary Health Care. 1992; 10 (2): 128-133. doi: 10.3109/02813439209014049.

[11] Wingard DL, Jones DW, Kaplan RM. Institutional care utilization by the elderly: a critical review. The Gerontologist. 1987; 27 (2): 156-163. doi: 10.1093/geront/27.2.156.

[12] Yaffe K, Patrick F, Newcomer R, et al. Patient and caregiver characteristics and nursing home placement in patients with dementia. JAMA. 2002; 287 (16): 2090-2097. doi: 10.1001/jama.287.16.2090.

[13] Heyman A, Peterson B, Fillenbaum G, Pieper C. Predictors of time to institutionalization of patients with Alzheimer's disease: the CERAD experience, part XVII. Neurology. 1997; 48 (5): 1304-1309. doi: 10.1212/wnl.48.5.1304.

[14] Knopman DS, Kitto J, Deinard S, Heiring J. Longitudinal study of death and institutionalization in patients with primary degenerative dementia. Journal of the American Geriatrics Society. 1988; 36 (2): 108-112. doi: 10.1111/j.15325415.1988.tb01778.x.

[15] Knopman DS, Berg JD, Thomas R, Grundman M, Thal LJ, Sano M. Nursing home placement is related to dementia progression: experience from a clinical trial. Neurology. 1999; 52 (4): 714-718. doi: 10.1212/wnl.52.4.714

[16] Miller SC, Prohaska TR, Furner SE, Freels S, Brody JA, Levy PS. Time to nursing home admission for persons with Alzheimer's disease: the effect of health care system characteristics. The Journals of Gerontology Series B: Psychological Sciences and Social Sciences. 1998; 53 (6): S341-S353. doi: 10.1093/geronb/53b.6.s341.

[17] Severson MA, Smith GE, Tangalos EG, et al. Patterns and predictors of institutionalization in community-based dementia patients. Journal of the American Geriatrics Society. 1994; 42 (2): 181-185. doi: 10.1111/j.15325415.1994.tb04949.x.

[18] Stern Y, Tang MX, Albert MS, et al. Predicting time to nursing home care and death in individuals with Alzheimer disease. JAMA. 1997; 277 (10): 806-812. doi: 10.1001/jama.1997.03540340040030.

[19] Colerick EJ, George LK. Predictors of institutionalization among caregivers of patients with Alzheimer's disease. Journal of the American Geriatrics Society. 1986; 34 (7): 493498. doi: 10.1111/j.1532-5415.1986.tb04239.x.

[20] Fisher L, Lieberman MA. A longitudinal study of predictors of nursing home placement for patients with dementia: the contribution of family characteristics. The Gerontologist. 1999; 39 (6): 677-686. doi: 10.1093/geront/39.6.677.

[21] Gambassi G, Landi F, Lapane KL, Sgadari A, Mor V, Bernabei R. Predictors of mortality in patients with Alzheimer's disease living in nursing homes. Journal of Neurology, Neurosurgery \& Psychiatry. 1999; 67 (1): 59-65. doi: $10.1136 /$ jnnp.67.1.59. 
[22] Hjaltadóttir I, Hallberg IR, Ekwall AK, Nyberg P. Predicting mortality of residents at admission to nursing home: A longitudinal cohort study. BMC Health Services Research. 2011; 11 (1): 86. doi: 10.1186/1472-6963-11-86.

[23] Mitchell SL, Morris JN, Park PS, Fries BE. Terminal care for persons with advanced dementia in the nursing home and home care settings. Journal of Palliative Medicine. 2004; 7 (6): 808-816. doi: 10.1089/jpm.2004.7.808.

[24] Callahan CM, Arling G, Tu W, et al. Transitions in care for older adults with and without dementia. Journal of the American Geriatrics Society. 2012; 60 (5): 813-820. doi: 10.1111/j.1532-5415.2012.03905.x.

[25] Tang F, Lee Y. Home-and community-based services utilization and aging in place. Home Health Care Services Quarterly. 2010; 29 (3): 138-154. doi: $10.1080 / 01621424.2010 .511518$.

[26] Zarit SH, Gaugler JE, Jarrott SE. Useful services for families: research findings and directions. International Journal of Geriatric Psychiatry. 1999; 14 (3): 165-178. doi: 10.1002/(sici) 1099-1166 (199903) 14: $3<165:$ :aid-gps966 > 3.3.co; $2-\mathrm{f}$.

[27] Zarit SH, Stephens MAP, Townsend A, Greene R. Stress reduction for family caregivers: effects of adult day care use. The Journals of Gerontology Series B: Psychological Sciences and Social Sciences. 1998; 53B (5): S267-S278. doi: 10.1093/geronb/53b.5.s267.

[28] Gaugler JE, Kane RL, Kane RA, Newcomer R. Early community-based service utilization and its effects on institutionalization in dementia caregiving. The Gerontologist. 2005; 45 (2): 177-185. doi: 10.1093/geront/45.2.177.

[29] Luppa M, Luck T, Brahler E, Koenig HH, Riedel-Heller SG. Prediction of institutionalization in dementia. Dementia and Geriatric Cognitive Disorders. 2008; 26 (1): 65-78. doi: $10.1159 / 000144027$.

[30] Kelly R, Puurveen G, Gill R. The effect of adult day services on delay to institutional placement. Journal of Applied Gerontology. 2014; $35 \quad$ (8): 814-835. doi: $10.1177 / 0733464814521319$.

[31] Gaugler JE, Zarit SH. The effectiveness of adult day services for disabled older people. Journal of Aging \& Social Policy. 2001; 12 (2): 23-47. doi: 10.1300/j031v12n02_03.

[32] McKhann G, Drachman D, Folstein M, Katzman R, Price D, Stadlan EM. Clinical diagnosis of Alzheimer's disease: report of the NINCDS-ADRDA work group* under the auspices of Department of Health and Human Services Task Force on Alzheimer's disease. Neurology. 1984; 34 (7): 939. doi: 10.1212/wnl.34.7.939.

[33] Doody RS, Pavlik V, Massman P, Rountree S, Darby E, Chan W. Predicting progression of Alzheimer's disease. Alzheimer's Research \& Therapy. 2010; 2 (2): 1-9. doi: 10.1186/alzrt25.
[34] Folstein MF, Folstein SE, McHugh PR. "Mini-mental state": a practical method for grading the cognitive state of patients for the clinician. Journal of Psychiatric Research. 1975; 12 (3): 189-198. doi: 10.1016/0022-3956 (75) 90026-6.

[35] Doody RS, Dunn JK, Huang E, Azher S, Kataki M. A method for estimating duration of illness in Alzheimer's disease. Dementia and Geriatric Cognitive Disorders. 2004; 17 (1-2): 1-4. doi: 10.1159/000074078.

[36] Lawton MP, Brody EM. Assessment of older people: selfmaintaining and instrumental activities of daily living. The Gerontologist. 1969; 9 (3): 179-186. doi: 10.1093/geront/9.3_part_1.179.

[37] SAS software. Cary, NC: SAS Institute Inc.; 2014.

[38] Wattmo C, Wallin ÅK. Early-versus late-onset Alzheimer disease: long-term functional outcomes, nursing home placement, and risk factors for rate of progression. Dementia and Geriatric Cognitive Disorders Extra. 2017; 7 (1): 172187. doi: $10.1159 / 000455943$.

[39] Takahashi PY, Tung EE, Crane SJ, Chaudhry R, Cha S, Hanson GJ. Use of the elderly risk assessment (ERA) index to predict 2-year mortality and nursing home placement among community dwelling older adults. Archives of Gerontology and Geriatrics. 2012; 54 (1): 34-38. doi: 10.1016/j.archger.2011.02.012.

[40] Rountree SD, Chan W, Pavlik VN, Darby EJ, Doody RS. Factors that influence survival in a probable Alzheimer disease cohort. Alzheimer's Research \& Therapy. 2012; 4 (3): 1-6. doi: 10.1186/alzrt119.

[41] Powers M. The community's emerging role in value-based health and social services. Generations. 2018; 42 (1): 4-8.

[42] Subira B. The value of the hospital-CBO partnerships in achieving the triple aim. Generations. 2018; 42 (1): 9-13.

[43] Karlin BE, Visnic S, McGee JS, Teri L. Results from the multisite implementation of STAR-VA: a multicomponent psychosocial intervention for managing challenging dementiarelated behaviors of veterans. Psychological Services. 2014; 11 (2): 200-208. doi: 10.1037/a0033683.

[44] Wattmo C, Wallin AK, Londos E, Minthon L. Risk factors for nursing home placement in Alzheimer's disease: a longitudinal study of cognition, ADL, service utilization, and cholinesterase inhibitor treatment. The Gerontologist. 2010; 51 (1): 17-27. doi: 10.1093/geront/gnq050.

[45] Doody RS, Massman P, Dunn JK. A method for estimating progression rates in Alzheimer disease. Archives of Neurology. 2001; 58 (3): 449-454. doi: 10.1001/archneur.58.3.449.

[46] Doody R, Pavlik V, Massman P, et al. Changing patient characteristics and survival experience in an Alzheimer's center patient cohort. Dementia and Geriatric Cognitive Disorders. 2005; 20 (2): 198-208. doi: 10.1159/000087300. 